\title{
Computational Analysis and Simulation of Vacuum Infusion Molding Process
}

\author{
Young Seok Song ${ }^{1}$ and Taikyeong T. Jeong ${ }^{2, \star}$ \\ ${ }^{1}$ Center for Composite Materials \\ University of Delaware, Newark, DE 19716 USA \\ youngseoks@gmail. com \\ ${ }^{2}$ Department of Electrical and Computer Engineering \\ University of Delaware, Newark, DE 19716 USA \\ ttjeong@alumni.utexas.net
}

\begin{abstract}
The current work focuses on resin bleeding process during the Seeman composite resin infusion molding process (SCRIMP), which is a subset of liquid composite molding (LCM) process. Finite difference method (FDM) is implemented to predict the preform thickness, bleeding resin volume, and fiber volume fraction by using a non-rigid control volume. After the fibrous preform is completely impregnated, the resin flow within the preform has a great impact on the dimension and mechanical properties of the final composite parts. As the resin flows out of the preform, the resin pressure and preform thickness are reduced, which increases the fiber volume fraction and the dimension tolerance of the preform.

In this paper, the influence of resin flow rate at vent in the mold is also investigated. It is found that there is a critical flow rate to optimize the SCRIMP process at the vacuum line.
\end{abstract}

\section{Introduction}

The SCRIMP is a variant of the traditional resin transfer molding (RTM) process, which is known to be suitable for processing large-scale composite structures [1, 2, 3]. The process can be explained as follows. The dry preform is laid up and covered with a very high permeable media. A peel ply is laid in between them. All of the layers are then sealed in a plastic film/bag and then vacuum is applied to evacuate entrapped air. When the resin inlet line is opened the resin is driven by atmosphere pressure through the infusion line until the preform is fully impregnated and the resin is cured. Since the process uses a single-sided tool, room temperature resin and vacuum only, SCRIMP shows great potential ability to form large-scale composites parts [4, [5, 6].

The resin bleeding stage of SCRIMP is from the time when preform is fully infused to the time when resin is completely cured. The flow behavior of resin during this period will significantly affect the dimensional tolerance, fiber volume fraction and the void content of the composite. Spatial variation in thickness

\footnotetext{
* T. Jeong is the corresponding author.
} 
and fiber volume fraction influences the mechanical properties of the composite laminate. In the SCRIMP process, resin is infused by opening the infusion line. During the infusion process, there exists a pressure gradient along the preform from the infusion line to the vacuum line. Since the sealed preform is not confined by the mold on top, the resin pressure gradient should cause a thickness gradient of the preform, which will be frozen in the cured final composite part. SCRIMP processing can be described as two different stages. The first stage is the infusion stage, which ends when the preform is infused completely. The second stage is the post-infusion stage, which starts after the first stage and ends when the resin is completely cured. Much attention has been paid on the first stage and much research has been done [6], 7], 8], [9] [10, to understand the resin flow and saturation behavior and to fulfill a proper infusion strategy. When the infusion is complete, therefore, one generally closes the infusion line or keeps resin circulating till the resin cures. It seems that the entire SCRIMP process has been complete by the end of the first stage, however, during the post-infusion stage, resin flow, pressure gradient, thickness gradient and the rheological properties of the resin continue changing as a function of time and location. These changes will finally determine the dimensional tolerance, fiber volume fraction; void content and mechanical properties of the composite.

In this paper, SCRIMP process is investigated by using FDM. We, particularly, establish a computational model for resin bleeding process in SCRIMP to analyze the resin flow behavior and examine the effects of different processing parameters, resin and fabric properties on the change of laminate thickness and fiber volume fraction.

\section{Description of Resin Bleeding Process}

\subsection{Governing Equations}

Darcy's law can be used as the basic momentum governing equation. For onedimensional problem, it can be expressed as follows.

$$
\mu_{x}=\frac{Q_{x}}{A}=-\frac{K}{\mu} \cdot \frac{d P}{d x}
$$

where $\mu_{x}$ is the flow velocity in x-axes and $\mathrm{A}$ is the cross-sectional area of preform. $\mathrm{K}$ is the effective permeability of the preform, $\mu$ is the viscosity of resin, and $\frac{d P}{d x}$ is the pressure gradient of the resin. When the filled mold is rigid, the one-dimensional equation of mass conservation for the fluid can be written as.

$$
\frac{d \mu_{x}}{d x}=0
$$

Substituting equation (1) into equation (2) leads to:

$$
\frac{d}{d x}\left(\frac{K}{\mu} \cdot \frac{d P}{d x}\right)=0
$$


This equation implies a linear pressure distribution between the resin infusion line and the flow front. If the resin pressure at the infusion line is $P_{0}$, the pressure distribution would have of the following form.

$$
P=P_{0}\left(1-\frac{x}{x_{f}}\right)=0
$$

where $x_{f}$ is the location of the flow front.

During the SCRIMP process, the preform is compressed by the atmospheric pressure and there is no rigid mold on top of the preform. Therefore the resin pressure gradient will cause the thickness variation across the preform. Since the preform for SCRIMP is not rigid, the resin pressure distribution may deviate from equation (4). However, when the preform is fully infused, the thickness will not change if one circulates the resin. At that situation, the preform can be approximately considered rigid and the resin pressure can be expressed by equation (4).

The flow behavior of resin and thickness change during the post-infusion period is related to different processing scenarios and parameters. After the preform is infused completely, if one circulates the resin until cured, a pressure gradient in the part will remain the same as that during the infusion period and thus the thickness gradient will be frozen into the part after cure. So, measures usually need to be taken before the resin is cured.

SCRIMP process can be assumed as compressible in the thickness direction and incompressible in the in-plane directions. A one-dimensional semi-rigid control volume of the preform after complete infusion is employed in the current study. We obtain equation (5), from the mass conservation principle.

$$
Q_{\text {in }}-Q_{\text {out }}=d v
$$

where $Q_{\text {in }}$ is the flow rate entering the control volume and $Q_{\text {out }}$ is the flow rate out of the control volume. dv denotes the rate of volume change of the control volume. By using Darcy's law, flow rates in and out to the control volume can be expressed as.

$$
\begin{aligned}
Q_{\text {in }} & =-\frac{K}{\mu} \frac{\partial P(x, t)}{\partial x} A_{\text {in }}=-\frac{K}{\mu} \frac{\partial P(x, t)}{\partial x} W \varepsilon(x, t) \\
Q_{\text {out }} & =-\frac{K}{\mu} \frac{\partial P(x+d x, t)}{\partial x} A_{\text {out }}=-\frac{K}{\mu} \frac{\partial P(x+d x, t)}{\partial x} W \varepsilon(x+d x, t) \\
& \approx-\frac{K}{\mu} \frac{\partial P(x, t)}{\partial x} W \varepsilon(x, t)+\frac{\partial}{\partial x}\left[-\frac{K}{\mu} \frac{\partial P(x, t)}{\partial x} W \cdot \varepsilon(x, t)\right] \cdot d x \\
d v & =\frac{\partial \varepsilon(x, t)}{\partial t} \cdot W \cdot \varepsilon(x, t)
\end{aligned}
$$

where $W$ is the width, $\varepsilon$ is the thickness of preform, and $t$ is the time. After substituting equation (6), (7), and (8) into equation (5), we finally obtain

$$
\frac{\partial}{\partial x}\left[-\frac{K}{\mu} \cdot \frac{\partial P(x, t)}{\partial x} \cdot W \cdot \varepsilon(x, t)\right]=\frac{\partial \varepsilon(x, t)}{\partial t}
$$


The thickness of the preform at any time can be expressed as

$$
\varepsilon(x, t)=f(P(x, t))
$$

Researchers have modeled the relation between preform thickness and resin pressure both empirically and analytically. We have used a fourth order polynomial empirical equation as our compaction model. Equation (9) can then be rewritten as

$$
\frac{\partial}{\partial x}\left[-\frac{K}{\mu} \cdot \frac{\partial P(x, t)}{\partial x} \cdot W \cdot f(P(x, t))\right]=\frac{\partial f(P(x, t))}{\partial t}
$$

\subsection{Computational Scheme}

When the infusion line is closed, there is no resin entering into the preform. At the boundary surface $x=0$, the flow rate entering a control volume $Q_{i n}=0$. The flow rate of resin out of the control volume is equal to the rate of change of volume.

$$
\frac{K}{\mu} \frac{\partial P(x, t)}{\partial x} \cdot \varepsilon(x, t)=\frac{\varepsilon(x, t)}{\partial t} \cdot d x
$$

On the other end, $x=L$, resin flows out through a tube. Considering the control volume at the end, the flow rate into the control volume is

$$
Q_{\text {in }}=-\frac{K}{\mu} \cdot \frac{\partial P(x, t)}{\partial x} \cdot W \cdot \varepsilon(x, t)
$$

The flow rate out of the volume is actually the flow rate in the tube and can be expressed as

$$
Q_{\text {out }}=Q_{\text {tube }}=\frac{\pi \cdot a^{4}}{8 \mu \cdot L_{0}} \cdot P(x, t)
$$

where $a$ is the radius of the tube, $L_{0}$ is the length of the tube, and $\mu$ is the viscosity of the resin. Therefore the boundary condition can be expressed as,

$$
-\frac{K}{\mu} \frac{\partial P(x, t)}{\partial x} \cdot \varepsilon(x, t)-\frac{H P(x, t)}{W}=\frac{\partial \varepsilon(x, t)}{\partial t} \cdot d x
$$

where $H=\frac{\pi \cdot a^{4}}{8 \mu \cdot L_{0}}$. At the moment when infusion line is closed $(\mathrm{t}=0)$, the resin pressure can be derived from the one dimensional Darcy's law and conservation principle. It is well known that the permeability of the preform is determined by the structure and fiber volume fraction of the preform. Since the thickness of the preform changes with time and location during the process, therefore, the permeability is also a function of time and location. The following equation is the relationship between permeability and fiber volume fraction.

$$
K=\kappa \frac{\left(1-V_{f}\right)^{3}}{V_{f}^{2}}
$$




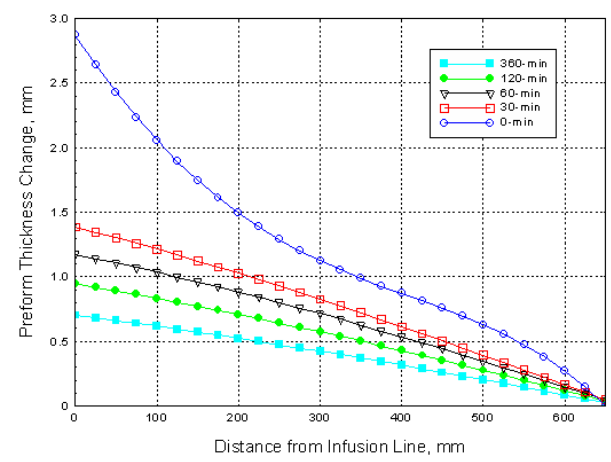

Fig. 1. Preform thickness change as a function of location (vacuum tube radius a $=2$ $\mathrm{mm}$ )

where $\kappa$ is the Kozeny Carman constant and $V_{f}$ is the fiber volume fraction. One-dimensional central finite difference method was employed to implement the numerical analysis. The detail description of finite difference scheme is shown in Figure 1. The length of preform was divided into $\mathrm{m}$ segments and time was also divided into small steps. Using the difference scheme presented, equation (9) can be discretized as

$$
\begin{gathered}
\frac{\partial}{\partial x}\left[\frac{K}{\mu} \cdot \frac{\partial P(x, t)}{\partial x} \cdot \varepsilon(x, t)\right] \approx \frac{K_{i}^{n}}{\mu^{n}} \cdot\left(\frac{\varepsilon_{i}^{n} \cdot \frac{P_{i+1}^{n}-P_{i}^{n} \triangle x}{\triangle x}-\varepsilon_{i-1}^{n} \cdot \frac{P_{i}^{n}-P_{i-1}^{n} \triangle x}{\triangle x}}{\triangle x}\right) \\
\frac{\partial \varepsilon(x, t)}{\partial t}=\frac{\varepsilon_{i}^{n}-\varepsilon_{i-1}^{n}}{\triangle t}
\end{gathered}
$$

Then, equation (9) can be recast as

$$
\varepsilon_{i}{ }^{n}+1=r_{i}^{n} \cdot\left[\varepsilon_{i}{ }^{n} \cdot\left(P_{i+1}^{n}-P_{i}^{n}\right)-\varepsilon_{i-1}^{n} \cdot\left(P_{i}^{n}-P_{i-1}^{n}\right)\right]+\varepsilon_{i}{ }^{n}
$$

where $r_{i}^{n}=\frac{K_{i}^{n}}{\mu^{n}} \cdot \frac{\Delta t}{\triangle x^{2}}$.

\section{Results and Discussion}

Figure 1 shows the preform thickness changes as a function of distance from infusion line at different time during the post-infusion period in the case of vacuum tube radius, a, of $2 \mathrm{~mm}$. The origin point is the infusion line position and the point $x=650 \mathrm{~mm}$ is the position of the vacuum line. It can be seen that the thickness at the infusion side decreased and that at vacuum side increased with time. As time passed by, the thickness gradient became smaller and finally became almost uniform after 6 hours when the gel point of the resin is reached. In the case of the tube radius is larger than $2 \mathrm{~mm}$, the thickness at vacuum side not changed with time. The decrease of thickness gradient was only caused by the decrease of thickness at the infusion side. 


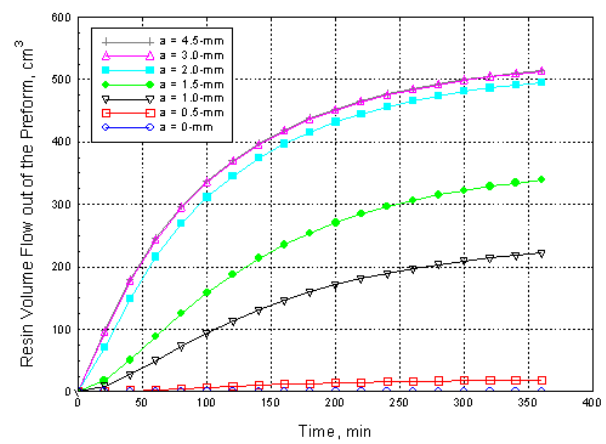

Fig. 2. Resin volume flowing out of the preform as a function of time with respect to various tube diameters, from $0 \mathrm{~mm}$ to $4.5 \mathrm{~mm}$

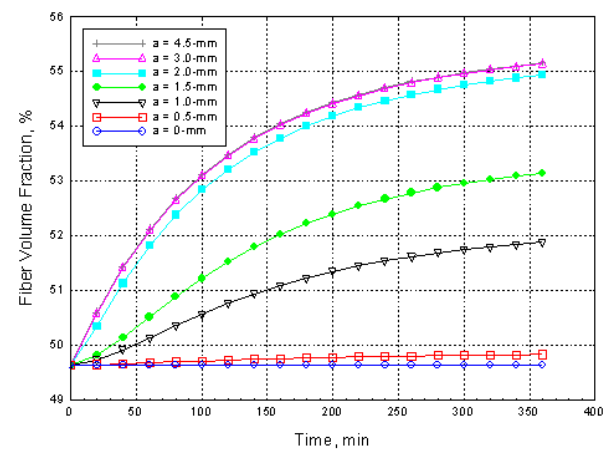

Fig. 3. Fiber volume fraction as a function of time with respect to various tube diameters, from $0 \mathrm{~mm}$ to $4.5 \mathrm{~mm}$

Figure 2 presents the resin volume flowing out of preform during post-infusion and Figure 3 is the fiber volume fraction change with time when the various tube diameters (from $0 \mathrm{~mm}$ to $4.5 \mathrm{~mm}$ ) are applied. It can be seen that larger vacuum tube sucked out more resin from the perform and the panel reached higher fiber volume fraction. However, excessive loss of resin before gel point may lead to voids in the composite laminate. Uniform thickness, high fiber volume fraction and low void content are the objectives for SCRIMP processing. Using the analysis presented above, we can balance and optimize these properties by choosing the proper radius of vacuum tube.

\section{Conclusion}

The post-infusion stage of the SCRIMP process significantly affects the dimensional tolerance, fiber volume fraction, and the bleeding resin volume. FDM is implemented to predict the preform thickness, bleeding resin volume, and fiber 
volume fraction by using a non-rigid control volume. As the resin flows out of the preform, the resin pressure and preform thickness are reduced, which increases the fiber volume fraction and the dimension tolerance of the preform. In this paper, the influence of resin flow rate at vent in the mold is also discussed. This work provides a practical tool to optimize the SCRIMP processing and the final composite properties.

\section{Acknowledgment}

The author would like to thank the referees for their valuable comments.

\section{References}

1. S. M. Lewit, J. C. Jakubowski, "Low cost VARTM process for commercial and military applications," 42nd International SAMPE Symposium, vol. 42, pp. 1173-1187, May 4-8, 1997

2. L. Joubaud, V. Achim, F. Trochu, "Numerical simulation of resin infusion and reinforcement consolidation under flexible cover," Polym. Compos., vol. 26, pp. 417-427, 2005

3. Y. S. Song, K. Chung, T. J. Kang, J. R. Youn, "Prediction of permeability tensor for three dimensional circular braided preform by applying a finite volume method to a unit cell," Compos. Sci. Technol., vol. 64, pp. 1629-1636, 2004

4. S. W. Beckwith, C. R. Hyland, "Resin transfer molding: a decade of technology advances," SAMPE Journal, vol. 34, pp. 7-19, 1998

5. M. K. Kang, W. I. Lee, H. T. Hahn, "Analysis of vacuum bag resin transfer molding process," Phys. Lett. A, vol. 329, pp. 207-213, 2004

6. T. G. Gotowski, G. Dillon, "The elastic deformation of fiber bundles, Advanced composite manufacturing, " New York John Wiley ES Sons, 1997

7. R. Mathur, D. Heider, C. Hoffmann, J. W. Gillespie, S. G. Advani, B. K. Fink, "Flow front measurements and model validation in the vacuum assisted resin transfer molding process," Polym. Compos., vol. 22, pp. 477-490, 2001

8. Y. Song, W. Chui, J. Glimm, B. Lindquist, F. Tangerman, "Applications of front tracking to the simulation of resin transfer molding," Computers Math. Applic., vol. 33 , pp. 47,1997

9. T. Jeong and A. Ambler, "Design trade-offs and power reduction techniques for high performance circuits and system," Springer-Verlag, ISSN: 0302-9743, 2006

10. J. Ni, Y. Zhao, L. J. Lee, S. Nakamura "Analysis of two-regional flow in liquid composite molding," Polym. Compos., vol. 18, pp. 254, 1997 